\title{
Maximal Repetition and Zero Entropy Rate
}

\author{
Łukasz Dębowski*
}

\begin{abstract}
Maximal repetition of a string is the maximal length of a repeated substring. This paper investigates maximal repetition of strings drawn from stochastic processes. Strengthening previous results, two new bounds for the almost sure growth rate of maximal repetition are identified: an upper bound in terms of conditional Rényi entropy of order $\gamma>1$ given a sufficiently long past and a lower bound in terms of unconditional Shannon entropy $(\gamma=1)$. Both the upper and the lower bound can be proved using an inequality for the distribution of recurrence time. We also supply an alternative proof of the lower bound which makes use of an inequality for the expectation of subword complexity. In particular, it is shown that a power-law logarithmic growth of maximal repetition with respect to the string length, recently observed for texts in natural language, may hold only if the conditional Rényi entropy rate given a sufficiently long past equals zero. According to this observation, natural language cannot be faithfully modeled by a typical hidden Markov process, which is a class of basic language models used in computational linguistics.
\end{abstract}

Keywords: maximal repetition, Rényi entropies, entropy rate, recurrence time, subword complexity, natural language

${ }^{*}$ Ł. Dębowski is with the Institute of Computer Science, Polish Academy of Sciences, ul. Jana Kazimierza 5, 01-248 Warszawa, Poland (e-mail: ldebowsk@ipipan.waw.pl). 


\section{Motivation and main results}

Maximal repetition $L\left(x_{1}^{n}\right)$ of a string $x_{1}^{n}=\left(x_{1}, x_{2}, \ldots, x_{n}\right)$ is the maximal length of a repeated substring. Put formally,

$$
L\left(x_{1}^{n}\right):=\max \left\{k: x_{i+1}^{i+k}=x_{j+1}^{j+k} \text { for some } 0 \leq i<j \leq n-k\right\} .
$$

Maximal repetition has been studied by computer scientists 1, 2, 3, 4, probabilists [5, 6, 7, 8, and information theorists [9, 10, 11]. Maximal repetition $L\left(x_{1}^{n}\right)$ can be computed efficiently for relatively long strings, in time $O(n)$ [2, which opens way to various empirical statistical studies. Moreover, for an arbitrary stochastic process $\left(X_{i}\right)_{i=-\infty}^{\infty}$, maximal repetition $L\left(X_{1}^{n}\right)$ is an nondecreasing function of the string length $n$. In this paper, we will investigate the rate of growth of maximal repetition for some stochastic processes.

Our theoretical investigations are motivated by an application to statistical modeling of natural language. In a previous paper of ours [12, we have been interested in the growth rate of maximal repetition for texts in natural language. Investigating 35 texts in English, French, and German, we have found that a power-law logarithmic growth of maximal repetition,

$$
L\left(x_{1}^{n}\right) \approx A(\log n)^{\alpha},
$$

holds approximately with $\alpha \approx 3$. This empirical result seems rather significant. It should be immediately noted that for a random permutation of text characters, we observe the plain logarithmic growth of maximal repetition,

$$
L\left(x_{1}^{n}\right) \approx A \log n .
$$

For a quick reference, in Figure 1, we present the data for a collection of plays by William Shakespeare, downloaded from Project Gutenberg (http://www. gutenberg.org/). To smooth the plot, we have computed maximal repetition for strings $x_{c_{n}+1}^{c_{n}+n}$ rather than $x_{1}^{n}$, where offsets $c_{n}$ are selected at random.

Consequently, we may ask what the empirical law (2) can tell us about the stochastic mechanism of natural language generation. Let $\left(X_{i}\right)_{i=-\infty}^{\infty}$ be a stationary process. We consider the Shannon entropy

$$
H(n):=\mathbf{E}\left[-\log P\left(X_{1}^{n}\right)\right]
$$

and the associated Shannon entropy rate

$$
h:=\lim _{n \rightarrow \infty} \frac{H(n)}{n} .
$$

Maximal repetition $L\left(X_{1}^{n}\right)$ resembles another statistic that has been intensely investigated, the longest match length $L_{n}$, which is the maximal length $k$ such that string $X_{0}^{k-1}$ is a substring of $X_{-n}^{-1}[13,14,15,16$. As shown in [13, for a stationary ergodic process over a finite alphabet, we have the pointwise convergence

$$
\lim _{n \rightarrow \infty} \frac{\log n}{1+L_{n}}=h \text { a.s. }
$$




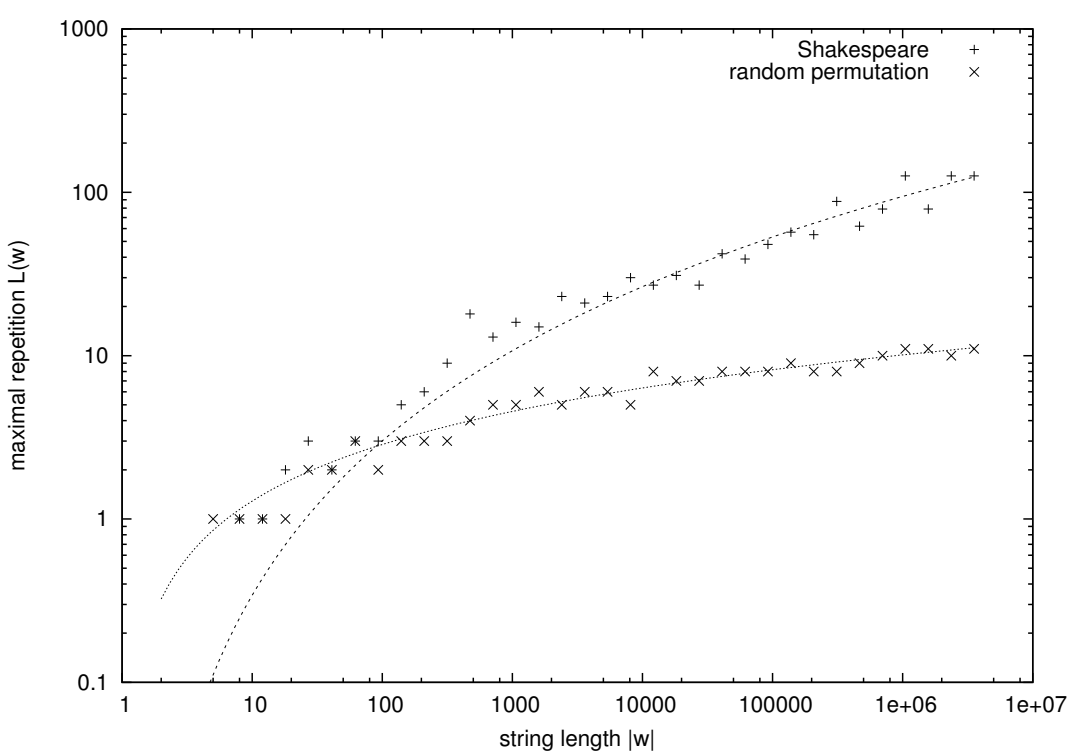

Figure 1: Maximal repetition for the concatenation of 35 plays by William Shakespeare and a random permutation of the text characters. To smooth the results, we sampled substrings $w=x_{c_{n}+1}^{c_{n}+n}$ of length $|w|=n$ from both sources at random assuming a uniform probability distribution on $c_{n}=0,1, \ldots, N-n$, where $N$ is the source length. For each length $|w|=n$, only one substrings $w=x_{c_{n}+1}^{c_{n}+n}$ was sampled. The fitted model is $L\left(x_{c_{n}+1}^{c_{n}+n}\right) \approx 0.02498(\log n)^{3.136}$ for Shakespeare and $L\left(x_{c_{n}+1}^{c_{n}+n}\right) \approx 0.4936(\log n)^{1.150}$ for the random permutation.

Since $L_{n} \leq L\left(X_{-n}^{n-1}\right)$ then, as discussed by Shields [7, we obtain a logarithmic bound for the maximal repetition,

$$
\limsup _{n \rightarrow \infty} \frac{\log n}{1+L\left(X_{-n}^{n-1}\right)} \leq h \text { a.s. }
$$

Hence the growth rate of maximal repetition provides a lower bound for the Shannon entropy rate.

Does then the power-law logarithmic growth of maximal repetition (2) imply that the Shannon entropy rate of natural language is zero? Here let us note that the overwhelming evidence collected so far suggests that the Shannon entropy rate of natural language is strictly positive, $h \approx 1$ bit per character [17, 18, 19, 20, 21, 22 but among researchers investigating this question there was an exception. Namely, Hilberg [23] supposed that the Shannon entropy of natural language satisfies condition $H(n) \approx B n^{\beta}$, where $\beta \approx 0.5$, and consequently the Shannon entropy rate might be zero. Although we have not been convinced that the Shannon entropy rate of natural language equals zero, for some time we have been interested in relaxations and strengthenings of Hilberg's hypothesis, see [10, 11, 12, 22]. In particular, we have been quite disturbed by the powerlaw logarithmic growth of maximal repetition for natural language, which we observed by the way. In [12, we supposed that it should be linked with vanishing of some sort of an entropy rate. 
This hypothetical entropy rate cannot be the Shannon entropy rate, however. As also shown by Shields [7, bound (7) is not tight. For any stationary ergodic process $\left(X_{i}\right)_{i=-\infty}^{\infty}$ and a function $\lambda(n)=o(n)$, there is a measurable function $f$ of infinite sequences and a stationary ergodic process $\left(Y_{i}\right)_{i=-\infty}^{\infty}$, where $Y_{i}:=$ $f\left(\left(X_{i+j}\right)_{j=-\infty}^{\infty}\right)$, such that

$$
\limsup _{n \rightarrow \infty} \frac{L\left(Y_{1}^{n}\right)}{\lambda(n)} \geq 1 \text { a.s. }
$$

Whereas the Shannon entropy rate of process $\left(Y_{i}\right)_{i=-\infty}^{\infty}$ is smaller than that of process $\left(X_{i}\right)_{i=-\infty}^{\infty}$, a careful analysis of the proof shows that the difference between the two can be made arbitrarily small, cf. e.g. 24. Moreover, if we take $\left(X_{i}\right)_{i=-\infty}^{\infty}$ to be an IID process, then process $\left(Y_{i}\right)_{i=-\infty}^{\infty}$ is mixing and very weak Bernoulli. Hence the power-law logarithmic growth of maximal repetition (2) does not imply that the Shannon entropy rate of natural language is zero or that natural language is not mixing.

In spite of this negative result, in this article, we will show that the powerlaw logarithmic growth of maximal repetition is naturally linked to a power-law growth of some generalized block entropies and vanishing of some generalized entropy rates. For simplicity let us consider a stationary process $\left(X_{i}\right)_{i=-\infty}^{\infty}$. For a parameter $\gamma \in(0,1) \cup(1, \infty)$, the block Rényi entropy [25] is defined as

$$
H_{\gamma}(n):=\frac{1}{1-\gamma} \log \sum_{x_{1}^{n}} P\left(X_{1}^{n}=x_{1}^{n}\right)^{\gamma} .
$$

For $\gamma \in\{0,1, \infty\}$, we define the block Rényi entropy as

$$
H_{\gamma}(n):=\lim _{\delta \rightarrow \gamma} H_{\delta}(n)
$$

Some special cases of $H_{\gamma}(n)$ are:

1. Hartley entropy $H_{0}(n)=\log \operatorname{card}\left\{x_{1}^{n}: P\left(X_{1}^{n}=x_{1}^{n}\right)>0\right\}$,

2. Shannon entropy $H_{1}(n)=H(n)=\mathbf{E}\left[-\log P\left(X_{1}^{n}\right)\right]$,

3. collision entropy $H_{2}(n)=-\log \mathbf{E} P\left(X_{1}^{n}\right)$,

4. min-entropy $H_{\infty}(n)=-\log \max _{x_{1}^{n}} P\left(X_{1}^{n}=x_{1}^{n}\right)$.

We have $H_{\gamma}(n) \geq H_{\delta}(n)$ for $\gamma<\delta$ and $H_{\gamma}(n) \leq \frac{\gamma}{\gamma-1} H_{\infty}(n)$ for $\gamma>1$.

In our problem, we will also deal with some conditional Rényi entropies given the infinite past. In the literature, a few alternative definitions of conditional Rényi entropy have been presented, cf. [26, 27. Here we will use yet another definition which is algebraically simpler and arises naturally in our application. For a parameter $\gamma \in(0,1) \cup(1, \infty)$, the conditional block Rényi entropy will be defined as

$$
H_{\gamma}^{\text {cond }}(n):=\frac{1}{1-\gamma} \log \mathbf{E} \sum_{x_{1}^{n}} P\left(X_{1}^{n}=x_{1}^{n} \mid X_{-\infty}^{0}\right)^{\gamma} .
$$

For $\gamma \in\{0,1, \infty\}$, we define the conditional block Rényi entropy as

$$
H_{\gamma}^{\text {cond }}(n):=\lim _{\delta \rightarrow \gamma} H_{\delta}^{\text {cond }}(n) .
$$


We note that for $\gamma>1$ the conditional block Rényi entropy can be written as

$$
H_{\gamma}^{\text {cond }}(n)=-\frac{1}{\gamma-1} \log \mathbf{E}\left[P\left(X_{1}^{n} \mid X_{-\infty}^{0}\right)\right]^{\gamma-1}
$$

and hence we obtain the conditional block min-entropy

$$
H_{\infty}^{\text {cond }}(n)=-\log \operatorname{ess} \sup P\left(X_{1}^{n} \mid X_{-\infty}^{0}\right) .
$$

By the Jensen inequality, we have $H_{\gamma}^{\text {cond }}(n) \geq H_{\delta}^{\text {cond }}(n)$ for $\gamma<\delta$ and $H_{\gamma}^{\text {cond }}(n) \leq H_{\gamma}(n)$. In contrast, we need not have $H_{\gamma}^{\text {cond }}(n) \leq \frac{\gamma}{\gamma-1} H_{\infty}^{\text {cond }}(n)$ for $\gamma>1$ (consider for instance $\gamma=2$ ). By another application of the Jensen inequality and by equality $h=\mathbf{E}\left[-\log P\left(X_{1} \mid X_{-\infty}^{0}\right)\right]$, we obtain the chain of inequalities

$$
H_{\infty}^{\text {cond }}(n) \leq H_{\gamma}^{\text {cond }}(n) \leq h n \leq H(n) \leq H_{0}(n), \quad \gamma>1 .
$$

Resuming, entropies $H_{0}(n)$ and $H_{\infty}^{\text {cond }}(n)$ are the largest one and the smallest one of the introduced entropies, respectively.

The above definitions can be partly generalized for nonstationary processes, as well. For an arbitrary (possibly nonstationary) process $\left(X_{i}\right)_{i=1}^{\infty}$ over a countable alphabet, we generalize the definition of the block Hartley entropy as

$$
H_{0}(n):=\log \operatorname{card}\left\{x_{1}^{n}: P\left(X_{m+1}^{m+n}=x_{1}^{n}\right)>0 \text { for some } m \geq 0\right\}
$$

and the conditional block min-entropy as

$$
H_{\infty}^{\text {cond }}(n):=-\log \sup _{m \geq 0} \max _{1}^{m+n} P\left(X_{m+1}^{m+n}=x_{m+1}^{m+n} \mid X_{1}^{m}=x_{1}^{m}\right) .
$$

As we can check easily, entropies $H_{0}(n)$ and $H_{\infty}^{\text {cond }}(n)$ coincide with the previous definitions for a stationary process.

Concerning the links between the generalized entropies and the maximal repetition, we will begin with two simple results which consolidate and generalize earlier observations from [8, 12] - and are stated in more generality for nonstationary processes. Namely, we will show that entropies $H_{0}(n)$ and $H_{\infty}^{\text {cond }}(n)$ provide an inverse sandwich bound for the investigated statistic of strings.

The first proposition bounds the maximal repetition below with the Hartley entropy. The smaller is the Hartley entropy, the larger is the maximal repetition.

Theorem 1 (cf. [12]) For an arbitrary process $\left(X_{i}\right)_{i=1}^{\infty}$ over a countable alphabet, if

$$
H_{0}(k) \leq B k^{\beta}
$$

for sufficiently large $k$ for certain $B>0$ and $\beta>0$ then

$$
L\left(X_{1}^{n}\right) \geq A(\log n)^{\alpha}
$$

for sufficiently large $n$ almost surely, for any $A<B^{-\alpha}$ and $\alpha=1 / \beta$.

Proof: Since the alphabet is countable, $P\left(X_{i+1}^{i+k}\right)>0$ holds almost surely for all $0 \leq i<\infty$. Hence block $X_{1}^{n}$ contains almost surely no more than $\exp \left(H_{0}(k)\right)$ different strings of length $k$. In particular if $\exp \left(H_{0}(k)\right)<n-k+1$ then block $X_{1}^{n}$ contains a repeat of length $k$, i.e., $L\left(X_{1}^{n}\right) \geq k$. Assume that $H_{0}(k) \leq B k^{\beta}$ holds for sufficiently large $k$. If we put $k_{n}=A(\log n)^{1 / \beta}$ where $A<B^{-1 / \beta}$ then we obtain $H_{0}\left(k_{n}\right) \leq B k_{n}^{\beta}<\log \left(n-k_{n}+1\right)$ for sufficiently large $n$. Hence $L\left(X_{1}^{n}\right) \geq k_{n}$ almost surely. 
Theorem 1 was proved in 12 for stationary processes. In 11, some stationary processes were constructed that satisfy both condition $H_{0}(n) \approx B n^{\beta}$ and condition $L\left(X_{1}^{n}\right) \approx A(\log n)^{\alpha}$ for an arbitrary $\beta$ and $\alpha=1 / \beta$.

In the second proposition we will bound the maximal repetition above with the conditional min-entropy. Before, let us make a simple observation that the conditional min-entropy is superadditive,

$$
H_{\infty}^{\text {cond }}(m+n) \geq H_{\infty}^{\text {cond }}(m)+H_{\infty}^{\text {cond }}(n) .
$$

Hence by the Fekete lemma, we have

$$
\lim _{n \rightarrow \infty} \frac{H_{\infty}^{\text {cond }}(n)}{n}=\sup _{n \geq 0} \frac{H_{\infty}^{\text {cond }}(n)}{n}
$$

and consequently this limit equals zero if and only if $H_{\infty}^{\text {cond }}(n)=0$ for all $n$. Now, we observe that the larger is the conditional min-entropy, the smaller is the maximal repetition.

Theorem 2 (cf. [8]) For an arbitrary process $\left(X_{i}\right)_{i=1}^{\infty}$ over a countable alphabet, if

$$
H_{\infty}^{\text {cond }}(k) \geq B k
$$

for sufficiently large $k$ for a certain $B>0$ then

$$
L\left(X_{1}^{n}\right)<A \log n
$$

for sufficiently large $n$ almost surely, for any $A>3 B^{-1}$.

Proof: We have

$$
\begin{aligned}
P\left(L\left(X_{1}^{n}\right) \geq k\right) & =P\left(X_{i+1}^{i+k}=X_{j+1}^{j+k} \text { for some } 0 \leq i<j \leq n-k\right) \\
& \leq \sum_{0 \leq i<j \leq n-k} P\left(X_{i+1}^{i+k}=X_{j+1}^{j+k}\right) \\
& =\sum_{0 \leq i<j \leq n-k} \sum_{x_{1}^{j}} P\left(X_{1}^{j}=x_{1}^{j}\right) P\left(X_{j+1}^{j+k}=x_{i+1}^{i+k} \mid X_{1}^{j}=x_{1}^{j}\right) \\
& \leq \sum_{0 \leq i<j \leq n-k} \exp \left(-H_{\infty}^{c o n d}(k)\right) \leq n^{2} \exp \left(-H_{\infty}^{c o n d}(k)\right) .
\end{aligned}
$$

Assume now that $H_{\infty}^{\text {cond }}(k) \geq B k$ holds for sufficiently large $k$. If we put $k_{n}=A \log n$ then we obtain

$$
\sum_{n=1}^{\infty} P\left(L\left(X_{1}^{n}\right) \geq k_{n}\right) \leq \sum_{n=1}^{\infty} n^{2} \exp \left(-H_{\infty}^{\text {cond }}\left(k_{n}\right)\right) \leq C+\sum_{n=1}^{\infty} n^{2-B A},
$$

which is finite if $A>3 B^{-1}$. Hence by the Borel-Cantelli lemma, we obtain that $L\left(X_{1}^{n}\right)<A \log n$ for sufficiently large $n$ almost surely.

Inequality 23) was demonstrated in [8, using a somewhat complicated technique involving source coding, for processes satisfying the equivalent finite energy condition

$$
P\left(X_{m+1}^{m+n}=x_{m+1}^{m+n} \mid X_{1}^{m}=x_{1}^{m}\right) \leq K c^{n}
$$


where $K>0$ and $0<c<1$. Condition (26) appears intuitive. We would expect it from well-behaved processes. In fact, finite energy processes include typical hidden Markov processes, uniformly dithered processes, processes satisfying the Doeblin conditions, as well as nonatomic $\psi$-mixing processes.

For clarity and completeness, let us state the respective results formally. A discrete process $\left(Y_{i}\right)_{i=-\infty}^{\infty}$ is called a hidden Markov process if $Y_{i}=f\left(X_{i}\right)$ for a certain function $f$ and a discrete Markov process $\left(X_{i}\right)_{i=-\infty}^{\infty}$.

Theorem 3 For a stationary hidden Markov process $\left(Y_{i}\right)_{i=-\infty}^{\infty}$ let the underlying Markov process be $\left(X_{i}\right)_{i=-\infty}^{\infty}$. Process $\left(Y_{i}\right)_{i=-\infty}^{\infty}$ is finite energy if

$$
c:=\sup _{y, x} P\left(Y_{i}=y \mid X_{i-1}=x\right)<1 .
$$

Proof: By conditional independence of $Y_{m+1}$ and $Y_{1}^{m}$ given $X_{m}$,

$$
\begin{aligned}
& P\left(Y_{m+1}=y_{m+1} \mid Y_{1}^{m}=y_{1}^{m}\right) \\
& =\sum_{x_{m}} P\left(Y_{m+1}=y_{m+1} \mid X_{m}=x_{m}\right) P\left(X_{m}=x_{m} \mid Y_{j_{1}^{m}}=y_{1}^{m}\right) \\
& \leq \sum_{x_{m}} c P\left(X_{m}=x_{m} \mid Y_{1}^{m}=y_{1}^{m}\right)=c .
\end{aligned}
$$

Thus process $\left(Y_{i}\right)_{i=-\infty}^{\infty}$ is finite energy.

Another subclass of finite energy processes are uniformly dithered processes, which generalize a construction by Shields $[8]$. Let $(\mathbb{X}, *)$ be a group. A stochastic process $\left(X_{i}\right)_{i=-\infty}^{\infty}$ over the alphabet $\mathbb{X}$ is called uniformly dithered if it satisfies $X_{i}=W_{i} * Z_{i}$, where $\left(W_{i}\right)_{i=-\infty}^{\infty}$ is an arbitrary process over the alphabet $\mathbb{X}$ and $\left(Z_{i}\right)_{i=-\infty}^{\infty}$ is an independent IID process with $P\left(Z_{i}=a\right) \leq c<1$.

Theorem 4 ([12]) Any uniformly dithered process is a finite energy process.

Let us observe that for a stationary process, condition (26) is equivalent to

$$
P\left(X_{1}^{n}=x_{1}^{n} \mid X_{-\infty}^{0}\right) \leq K c^{n} \text { a.s. }
$$

by the martingale convergence. There are two related Doeblin conditions

$$
\begin{aligned}
& P\left(X_{r}=x_{r} \mid X_{-\infty}^{0}\right) \geq d \text { a.s. } \\
& P\left(X_{r}=x_{r} \mid X_{-\infty}^{0}\right) \leq D \text { a.s. }
\end{aligned}
$$

for some $r \geq 1$ and $0<d, D<1$, cf. [16, 15]. The first condition, can be satisfied for a finite alphabet only.

Theorem 5 If a process assuming more than one value satisfies condition (30) then it satisfies condition (31). Moreover, if a stationary process satisfies condition (31) then it is finite energy.

Proof: First, assume condition (30). Then obviously

$$
P\left(X_{r}=x_{r} \mid X_{-\infty}^{0}\right)=1-\sum_{x_{r}^{\prime} \neq x_{r}} P\left(X_{r}=x_{r}^{\prime} \mid X_{-\infty}^{0}\right) \leq 1-d=: D,
$$

so we obtain condition (31). Next, assume condition (31). Then

$$
P\left(X_{1}^{r}=x_{1}^{r} \mid X_{-\infty}^{0}\right) \leq P\left(X_{r}=x_{r} \mid X_{-\infty}^{0}\right) \leq D
$$

and, by stationarity, $P\left(X_{1}^{n}=x_{1}^{n} \mid X_{-\infty}^{0}\right) \leq D^{\lfloor n / r\rfloor} \leq D^{n / r-1}$, so 29 follows. 
Independently, in 9 , inequality (23) was established for stationary processes that satisfy condition (30).

The last subclass of finite energy processes which we are going to discuss are nonatomic $\psi$-mixing processes. For a stationary process $\left(X_{i}\right)_{i=-\infty}^{\infty}$ define

$$
\psi(n)=\sup _{i, j \geq 1} \operatorname{ess} \sup \left[\frac{P\left(X_{-j}^{0}, X_{n}^{n+i}\right)}{P\left(X_{-j}^{0}\right) P\left(X_{n}^{n+i}\right)}-1\right] .
$$

The process is called $\psi$-mixing if $\lim _{n \rightarrow \infty} \psi(n)=0$.

Theorem 6 A $\psi$-mixing stationary process $\left(X_{i}\right)_{i=-\infty}^{\infty}$ is finite energy if

$$
\lim _{n \rightarrow \infty} \operatorname{ess} \sup P\left(X_{1}^{n}\right)=0 .
$$

Proof: A stationary process $\left(X_{i}\right)_{i=-\infty}^{\infty}$ has been called simple mixing in [28] if

$$
P\left(X_{-j}^{0}, X_{n}^{n+i}\right) \leq K P\left(X_{-j}^{0}\right) P\left(X_{n}^{n+i}\right)
$$

for all $n, i, j \geq 1$ and a $K>0$. Obviously, any $\psi$-mixing process is simple mixing. It has been shown in 28, Corollary 4.4] that if a simple mixing process $\left(X_{i}\right)_{i=-\infty}^{\infty}$ satisfies $(35)$ then $P\left(X_{1}^{n}\right) \leq c^{n}$ for some $0<c<1$. In consequence, any such process has the finite energy property by condition 36 .

There is an interesting application of the above results to natural language. Although hidden Markov processes are some classical models in computational linguistics [29, 30, 31, their insufficiency as models of natural language was often claimed earlier, cf. [31. Using Theorems 2, 3, and 5 and the empirical observation of the power-law logarithmic growth of maximal repetition (2), we can provide a rigorous way of demonstrating that natural language is not a typical hidden Markov process, cf. a different approach to this question in [32, and does not even satisfy the Doeblin condition, contrary to an empirically unsupported assertion in [15. Simply, as we have stated in the previous paragraph, typical hidden Markov processes and processes satisfying the Doeblin condition are finite energy, whereas the power-law logarithmic growth (2) by Theorem 2 excludes the class of finite energy processes.

Let us come back to the main thread. In view of Theorems 1 and 2 , the hyperlogarithmic growth of maximal repetition can be connected to vanishing of the Hartley entropy rate and the conditional min-entropy, as follows,

$$
\begin{array}{r}
\limsup _{n \rightarrow \infty} \frac{H_{0}(n)}{n}=0 \Longrightarrow \liminf _{n \rightarrow \infty} \frac{L\left(X_{1}^{n}\right)}{\log n}=\infty \text { a.s., } \\
\limsup _{n \rightarrow \infty} \frac{L\left(X_{1}^{n}\right)}{\log n}=\infty \text { a.s. } \Longrightarrow H_{\infty}^{\text {cond }}(n)=0, \quad n \geq 1 .
\end{array}
$$

Since the difference between $H_{0}(n)$ and $H_{\infty}^{\text {cond }}(n)$ can be arbitrarily large, we can ask a question whether the gap between the upper bound and the lower bound for the maximal repetition can be narrowed. The natural step is to consider other generalized entropies.

Now we can present some strengthening of Theorems 1 and 2 , which constitutes the main result of this article. The first proposition bounds the maximal repetition below with the Shannon entropy. The smaller is the Shannon entropy, the larger is the maximal repetition. 
Theorem 7 For a stationary process $\left(X_{i}\right)_{i=-\infty}^{\infty}$ over a countable alphabet, if

$$
H(k) \leq B k^{\beta}
$$

for sufficiently large $k$ for certain $B>0$ and $\beta>0$ then

$$
L\left(X_{1}^{n}\right)>(\log n)^{\alpha}
$$

for sufficiently large $n$ almost surely, for any $\alpha<1 / \beta$.

In contrast, the second proposition bounds the maximal repetition above in terms of the conditional Rényi entropy of order $\gamma>1$ given a sufficiently long but finite past. For a stationary process $\left(X_{i}\right)_{i=-\infty}^{\infty}$ over a finite alphabet $\mathbb{X}$ and $\gamma>1$, let us write $N(n):=(\operatorname{card} \mathbb{X})^{n}$ and

$$
\tilde{H}_{\gamma}^{c o n d}(n):=-\frac{1}{\gamma-1} \log \mathbf{E}\left[P\left(X_{1}^{n} \mid X_{-N(n)}^{0}\right)\right]^{\gamma-1},
$$

where $H_{\gamma}(n) \geq \tilde{H}_{\gamma}^{\text {cond }}(n) \geq H_{\gamma}^{\text {cond }}(n)$ by the Jensen inequality. The larger is the entropy $\tilde{H}_{\gamma}^{\text {cond }}(n)$, the smaller is the maximal repetition.

Theorem 8 For a stationary process $\left(X_{i}\right)_{i=-\infty}^{\infty}$ over a finite alphabet and a $\gamma>1$, if

$$
\tilde{H}_{\gamma}^{c o n d}(k) \geq B k^{\beta}
$$

for sufficiently large $k$ for certain $B>0$ and $\beta>0$ then

$$
L\left(X_{1}^{n}\right)<A(\log n)^{\alpha}
$$

for sufficiently large $n$ almost surely, for any $A>\left[\gamma \cdot \frac{\gamma+1}{\gamma-1}\right]^{\alpha} B^{-\alpha}$ and $\alpha=1 / \beta$.

Thus, the hyperlogarithmic growth of maximal repetition can be connected to vanishing of the Shannon entropy rate and the conditional Rényi entropy rate, as follows,

$$
\begin{aligned}
& h=\lim _{n \rightarrow \infty} \frac{H(n)}{n}=0 \Longrightarrow \liminf _{n \rightarrow \infty} \frac{L\left(X_{1}^{n}\right)}{(\log n)^{\alpha}}=\infty \text { a.s., } \quad \alpha<1, \\
& \limsup _{n \rightarrow \infty} \frac{L\left(X_{1}^{n}\right)}{\log n}=\infty \text { a.s. } \Longrightarrow \liminf _{n \rightarrow \infty} \frac{\tilde{H}_{\gamma}^{\text {cond }}(n)}{n}=0, \quad \gamma>1 .
\end{aligned}
$$

As we have mentioned, the first implication was noticed in [7]. Theorem 7] supplements this observation for the power-law growth of Shannon entropy. The gap between entropies $H(n)$ and $\tilde{H}_{\gamma}^{c o n d}(n)$ can be still arbitrarily large. It remains an open question whether Theorems 7 and 8 can be sharpened further. Can entropies $H(n)$ and $\tilde{H}_{\gamma}^{c o n d}(n)$ be both replaced with the unconditional Rényi entropy $H_{\gamma}(n)$ of any order $\gamma>1$ ? We suppose that the answer is negative but the counterexamples seem difficult to construct.

To conclude the introduction, a few words are due about the proofs of our new results and their historical context. Both Theorems 7 and 8 can be proved using the probabilistic upper and lower bounds for recurrence times by Kontoyiannis 33 . The recurrence times are random distances between two occurrences of a particular string in the realization of a stationary process. Recurrence 
times are a classical topic in ergodic theory and information theory. Their fundamental links with probability and Shannon entropy rate have been established in $34,35,13$. Less recognized are their links with Rényi entropy [28. Recently, recurrence times have been also researched experimentally for natural language [36. Additionally, we can supply an alternative proof of Theorem 7 which applies subword complexity and an inequality by Dębowski [37, 38. The subword complexity of a string is a function which tells how many different substrings of a given length appear in the string. Subword complexity has been studied mostly from a combinatorial perspective [39, 40, 1, 41, 42, whereas its links with entropy have not been much researched.

The remaining parts of this paper are organized as follows. In Section II. we prove Theorem 8 , whereas in Section III] we demonstrate Theorem 7, whose discussion partly relies on the discussion of Theorem 8

\section{Proof of Theorem 8}

Our proof of Theorem 8 applies the concept of the recurrence time, which is a special case of the waiting time. The waiting time $R\left(x_{1}^{k}\right)$ is a random variable equal to the first position in the infinite random past $X_{-\infty}^{-1+k}=$ $\left(\ldots, X_{-3+k}, X_{-2+k}, X_{-1+k}\right)$ at which a copy of a finite fixed string $x_{1}^{k}=$ $\left(x_{1}, x_{2}, \ldots, x_{k}\right)$ appears,

$$
R\left(x_{1}^{k}\right):=\inf \left\{i \geq 1: X_{-i+1}^{-i+k}=x_{1}^{k}\right\} .
$$

A particular case of the waiting time is the recurrence time $R_{k}:=R\left(X_{1}^{k}\right)$, where we plug in the random block $X_{1}^{k}$. To bound the maximal repetition with conditional Rényi entropy, we first link the distribution of maximal repetition to the expectation of the recurrence time.

Lemma 1 For a stationary process $\left(X_{i}\right)_{i=-\infty}^{\infty}$ over a countable alphabet,

$$
\begin{aligned}
& P\left(L\left(X_{1}^{n}\right)<k\right) \leq \frac{\mathbf{E} \log R_{k}}{\log (n-k+1)}, \\
& P\left(L\left(X_{1}^{n}\right) \geq k\right) \leq(n-k+1)^{\gamma} \mathbf{E} R_{k}^{-\gamma+1}, \quad \gamma>1 .
\end{aligned}
$$

Proof: Let $T$ be the shift operation, $X_{i} \circ T=X_{i+1}$. We have

$$
\left(L\left(X_{-n+k-1}^{k}\right) \geq k\right)=\bigcup_{i=k}^{n}\left(R_{k} \circ T^{-i} \leq n-i+1\right) .
$$

Hence by stationarity and the Markov inequality,

$$
\begin{aligned}
& P\left(L\left(X_{1}^{n}\right)<k\right) \leq P\left(R_{k}>n-k+1\right) \leq \frac{\mathbf{E} \log R_{k}}{\log (n-k+1)}, \\
& P\left(L\left(X_{1}^{n}\right) \geq k\right) \leq(n-k+1) P\left(R_{k} \leq n-k+1\right) \leq(n-k+1)^{\gamma} \mathbf{E} R_{k}^{-\gamma+1} .
\end{aligned}
$$


Now let us introduce trimmed waiting and recurrence times

$$
\begin{aligned}
S\left(x_{1}^{k}\right) & :=\min \left\{R\left(x_{1}^{k}\right), N(k)\right\} \leq R\left(x_{1}^{k}\right), \\
S_{k} & :=\min \left\{R_{k}, N(k)\right\} \leq R_{k},
\end{aligned}
$$

where $N(k):=(\operatorname{card} \mathbb{X})^{k}$ and $\mathbb{X}$ is the alphabet of $X_{i}$. Subsequently, we have a bound for the distribution of the trimmed recurrence time in terms of conditional probability. This bound is inspired by a similar bound for the untrimmed recurrence time $R_{k}$ given by Kontoyiannis 33. The result of Kontoyiannis applied conditional probability given the infinite past. Here we reduce this infinite past to a finite context. The proof technique remains essentially the same.

Lemma 2 (cf. [33]) For a process $\left(X_{i}\right)_{i=-\infty}^{\infty}$ over a finite alphabet $\mathbb{X}$, for any $C>0$, we have

$$
P\left(S_{k} \leq \frac{C}{P\left(X_{1}^{k} \mid X_{-N(k)}^{0}\right)}\right) \leq C(1+k \log \operatorname{card} \mathbb{X}) .
$$

Proof: By the conditional Markov inequality, we have

$$
\begin{aligned}
P\left(S_{k} \leq \frac{C}{P\left(X_{1}^{k} \mid X_{-N(k)}^{0}\right)}\right) & \leq \mathbf{E} \mathbf{E}\left(\frac{C}{S_{k} P\left(X_{1}^{k} \mid X_{-N(k)}^{0}\right)} \mid X_{-N(k)}^{0}\right) \\
& =\mathbf{E} \sum_{x_{1}^{k}} \frac{C}{S\left(x_{1}^{k}\right)} .
\end{aligned}
$$

But for each $i \geq 1$ there is at most one string $x_{1}^{k}$ such that $R\left(x_{1}^{k}\right)=i$, so we have a uniform almost sure bound

$$
\sum_{x_{1}^{k}} \frac{C}{S\left(x_{1}^{k}\right)} \leq \sum_{i=1}^{N(k)} \frac{C}{i} \leq C\left(1+\int_{1}^{N(k)} \frac{1}{u} d u\right) \leq C(1+k \log \operatorname{cord} \mathbb{X}) .
$$

Having demonstrated the above two lemmas, we are in a position to prove Theorem 8. For $\gamma>1$, assume $\tilde{H}_{\gamma}^{\text {cond }}(k) \geq B k^{\beta}$ for sufficiently large $k$. Observe that for $0 \leq X \leq 1$ and $Y \geq 0$, we have $\mathbf{E} X \leq \mathbf{E} Y+P(X \geq Y)$. Specializing this to $0 \leq S_{k}^{-1} \leq 1$ and $P\left(X_{1}^{k} \mid X_{-N(k)}^{0}\right) \geq 0$, by 54 we obtain

$$
\begin{aligned}
\mathbf{E} R_{k}^{-\gamma+1} & \leq \mathbf{E} S_{k}^{-\gamma+1} \\
& \leq \min _{C>0}\left[C^{-\gamma+1} \mathbf{E}\left[P\left(X_{1}^{k} \mid X_{-N(k)}^{0}\right)\right]^{-\gamma+1}+P\left(S_{k} \leq \frac{C}{P\left(X_{1}^{k} \mid X_{-N(k)}^{0}\right)}\right)\right] \\
& \leq \min _{C>0}\left[C^{-\gamma+1} \exp \left(-(\gamma-1) \tilde{H}_{\gamma}^{\text {cond }}(k)\right)+C k(\log \operatorname{card} \mathbb{X}+1)\right] \\
& \leq 2\left[(\log \operatorname{card} \mathbb{X}+1) k \exp \left(-\tilde{H}_{\gamma}^{\text {cond }}(k)\right)\right]^{\frac{\gamma-1}{\gamma}}
\end{aligned}
$$

Hence by (48), we obtain for sufficiently large $k$ that

$$
P\left(L\left(X_{1}^{n}\right) \geq k\right) \leq 2 n^{\gamma}\left[(\log \operatorname{card} \mathbb{X}+1) k \exp \left(-\tilde{H}_{\gamma}^{c o n d}(k)\right)\right]^{\frac{\gamma-1}{\gamma}} .
$$


Let us take $k_{n}=A(\log n)^{1 / \beta}$. Then

$$
\sum_{n=1}^{\infty} P\left(L\left(X_{1}^{n}\right) \geq k_{n}\right) \leq C+2 \sum_{n=1}^{\infty} n^{\gamma}\left[(\log \operatorname{card} \mathbb{X}+1) A(\log n)^{1 / \beta} n^{-B A^{\beta}}\right]^{\frac{\gamma-1}{\gamma}},
$$

which is finite for $A>\left[\gamma \cdot \frac{\gamma+1}{\gamma-1}\right]^{1 / \beta} B^{-1 / \beta}$. Hence by the Borel-Cantelli lemma, we have $L\left(X_{1}^{n}\right)<A(\log n)^{1 / \beta}$ for all but finitely many $n$ almost surely. In this way we have proved Theorem 8 .

\section{Two proofs of Theorem 7}

We will present two proofs of Theorem 7 . The first one uses inequality (47) and the second bound for the recurrence time by Kontoyiannis [33.

Lemma 3 (cf. [33]) For a stationary process $\left(X_{i}\right)_{i=-\infty}^{\infty}$ over a countable alphabet, for any $C>0$, we have

$$
P\left(R_{k} \geq \frac{C}{P\left(X_{1}^{k}\right)}\right) \leq C^{-1} .
$$

Proof: Recalling the celebrated Kac theorem,

$$
\mathbf{E}\left(R_{k} \mid X_{1}^{k}\right)=\frac{1}{P\left(X_{1}^{k}\right)},
$$

cf. [34, we obtain by the conditional Markov inequality

$$
\begin{aligned}
P\left(R_{k} \geq \frac{C}{P\left(X_{1}^{k}\right)}\right) & =\mathbf{E} P\left(R_{k} \geq \frac{C}{P\left(X_{1}^{k}\right)} \mid X_{1}^{k}\right) \\
& \leq \mathbf{E}\left[\frac{P\left(X_{1}^{k}\right) \mathbf{E}\left(R_{k} \mid X_{1}^{k}\right)}{C}\right]=C^{-1} .
\end{aligned}
$$

Observe that $\mathbf{E} X \leq \int_{0}^{\infty} P(X \geq p) d p$. Hence, applying Lemma 3 to inequality (47), we obtain

$$
\begin{aligned}
P\left(L\left(X_{1}^{n}\right)<k\right) & \leq \frac{\mathbf{E} \log R_{k}}{\log (n-k+1)} \\
& =\frac{\mathbf{E}\left[-\log P\left(X_{1}^{k}\right)\right]+\mathbf{E}\left[\log R_{k} P\left(X_{1}^{k}\right)\right]}{\log (n-k+1)} \\
& \leq \frac{H(k)+\int_{0}^{\infty} P\left(R_{k} P\left(X_{1}^{k}\right) \geq e^{p}\right) d p}{\log (n-k+1)} \\
& \leq \frac{H(k)+\int_{0}^{\infty} e^{-p} d p}{\log (n-k+1)}=\frac{H(k)+1}{\log (n-k+1)} .
\end{aligned}
$$


Assume $H(k) \leq B k^{\beta}$ for sufficiently large $k$. For an $l>0$ and an $\epsilon>0$, let us take $k_{l}=2^{l}, m=l^{1+\epsilon}$, and $n_{l}=\exp \left(k_{l}^{\beta+\epsilon}\right)$. By inequality 63 for $k=k_{l}$, $m=m_{l}$, and $n=n_{l}$, we obtain

$$
\sum_{l=1}^{\infty} P\left(L\left(X_{1}^{n_{l}}\right)<k_{l}\right) \leq C+\sum_{l=1}^{\infty} \frac{B 2^{l \beta}+1}{\log \left(\exp \left(2^{l(\beta+\epsilon)}\right)-2^{l}+1\right)}<\infty .
$$

Hence by the Borel-Cantelli lemma, we have $L\left(X_{1}^{n}\right) \geq k_{l}$ for all but finitely many $l$ almost surely. Now for an arbitrary $n>0$, let us consider the maximal $l$ such that $n_{l} \leq n$. We have $L\left(X_{1}^{n}\right) \geq L\left(X_{1}^{n_{l}}\right)$ since $X_{1}^{n_{l}}$ is a substring of $X_{1}^{n}$, whereas

$$
k_{l}=\frac{1}{2} k_{l+1}=\frac{1}{2}\left(\log n_{l+1}\right)^{1 /(\beta+\epsilon)} \geq \frac{1}{2}(\log n)^{1 /(\beta+\epsilon)} .
$$

Hence $L\left(X_{1}^{n}\right) \geq \frac{1}{2}(\log n)^{1 /(\beta+\epsilon)}$ holds for all but finitely many $n$ almost surely. This completes the first proof of Theorem 7 .

The second proof of Theorem 7 will make make use of another concept, namely, the notion of subword complexity. Subword complexity $f\left(k \mid x_{1}^{n}\right)$ is a function which counts how many distinct substrings of length $k$ appear in a string $x_{1}^{n}$,

$$
f\left(k \mid x_{1}^{n}\right):=\operatorname{card}\left\{y_{1}^{k}: x_{i+1}^{i+k}=y_{1}^{k} \text { for some } 0 \leq i \leq n-k\right\} .
$$

To bound the maximal repetition in terms of Shannon entropy, we first relate the distribution of maximal repetition to the expected subword complexity. The following proposition strengthens Lemma 1.

Lemma 4 We have

$$
\begin{aligned}
& P\left(L\left(X_{1}^{n}\right)<k\right) \leq \frac{\mathbf{E} f\left(k \mid X_{1}^{n}\right)}{n-k+1}, \\
& P\left(L\left(X_{1}^{n}\right) \geq k\right) \leq n-k+1-\mathbf{E} f\left(k \mid X_{1}^{n}\right) .
\end{aligned}
$$

Proof: We have $f\left(k \mid X_{1}^{n}\right)=n-k+1$ if $L\left(X_{1}^{n}\right)<k$ and $f\left(k \mid X_{1}^{n}\right) \leq n-k$ if $L\left(X_{1}^{n}\right) \geq k$. Hence

$$
\begin{aligned}
& \mathbf{E} f\left(k \mid X_{1}^{n}\right) \geq(n-k+1) P\left(L\left(X_{1}^{n}\right)<k\right), \\
& \mathbf{E} f\left(k \mid X_{1}^{n}\right) \leq(n-k+1) P\left(L\left(X_{1}^{n}\right)<k\right)+(n-k) P\left(L\left(X_{1}^{n}\right) \geq k\right),
\end{aligned}
$$

from which the claims follow.

Subsequently, we have a bound for the expected subword complexity in terms of Shannon entropy. The following Lemma 5 is a variation of the results in [37, 38. Precisely, in 37] we have established inequality (74), whereas in 38. we have given a bound similar to 71 but for the number of nonoverlapping blocks rather than the overlapping ones.

Lemma 5 (cf. [37, 38]) For a stationary process $\left(X_{i}\right)_{i=-\infty}^{\infty}$ over a countable alphabet, for any $m \geq 1$,

$$
\frac{\mathbf{E} f\left(k \mid X_{1}^{n}\right)}{n-k+1} \leq \frac{1}{m}+\frac{\exp (m H(k))}{n-k+1} .
$$


Proof: We will use the identity

$$
f\left(k \mid X_{1}^{n}\right)=\sum_{w \in \mathbb{X}^{k}} \mathbf{1}\left\{\sum_{i=0}^{n-k} \mathbf{1}\left\{X_{i+1}^{i+k}=w\right\} \geq 1\right\} .
$$

Hence by the Markov inequality,

$$
\begin{aligned}
\mathbf{E} f\left(k \mid X_{1}^{n}\right) & =\sum_{w \in \mathbb{X}^{k}} P\left(\sum_{i=0}^{n-k} \mathbf{1}\left\{X_{i+1}^{i+k}=w\right\} \geq 1\right) \\
& \leq \sum_{w \in \mathbb{X}^{k}} \min \left[1, \mathbf{E}\left(\sum_{i=0}^{n-k} \mathbf{1}\left\{X_{i+1}^{i+k}=w\right\}\right)\right] \\
& =\sum_{w \in \mathbb{X}^{k}} \min \left[1,(n-k+1) P\left(X_{1}^{k}=w\right)\right] \\
& =(n-k+1) \mathbf{E}\left(\min \left(\left[(n-k+1) P\left(X_{1}^{k}\right)\right]^{-1}, 1\right)\right) .
\end{aligned}
$$

Denoting $\sigma(y)=\min [\exp (y), 1]$, we obtain

$$
\frac{\mathbf{E} f\left(k \mid X_{1}^{n}\right)}{n-k+1} \leq \mathbf{E} \sigma\left(-\log P\left(X_{1}^{k}\right)-\log (n-k+1)\right),
$$

where $\mathbf{E}\left[-\log P\left(X_{1}^{k}\right)\right]=H(k)$. Therefore, using the Markov inequality

$$
P\left(-\log P\left(X_{1}^{k}\right) \geq m H(k)\right) \leq \frac{1}{m}
$$

for $m \geq 1$, we further obtain from 74 that

$$
\frac{\mathbf{E} f\left(k \mid X_{1}^{n}\right)}{n-k+1} \leq \frac{1}{m}+\frac{\exp (m H(k))}{n-k+1} .
$$

Inserting (76) into 67) yields the requested bound.

The above two lemmas will be used now to demonstrate Theorem 7 Chaining inequalities (67) and (71), we obtain inequality

$$
P\left(L\left(X_{1}^{n}\right)<k\right) \leq \frac{1}{m}+\frac{\exp (m H(k))}{n-k+1},
$$

which resembles inequality (63). The sequel is essentially the same. Assume $H(k) \leq B k^{\beta}$ for sufficiently large $k$. For an $l>0$ and an $\epsilon>0$, let us take $k_{l}=2^{l}, m=l^{1+\epsilon}$, and $n_{l}=\exp \left(k_{l}^{\beta+\epsilon}\right)$. By inequality 77 for $k=k_{l}, m=m_{l}$, and $n=n_{l}$, we obtain

$$
\sum_{l=1}^{\infty} P\left(L\left(X_{1}^{n_{l}}\right)<k_{l}\right) \leq C+\sum_{l=1}^{\infty}\left[\frac{1}{l^{1+\epsilon}}+\frac{\exp \left(l^{1+\epsilon} B 2^{l \beta}\right)}{\exp \left(2^{l(\beta+\epsilon)}\right)-2^{l}+1}\right]<\infty .
$$

Hence by the Borel-Cantelli lemma, we have $L\left(X_{1}^{n}\right) \geq k_{l}$ for all but finitely many $l$ almost surely. Now for an arbitrary $n>0$, let us consider the maximal $l$ such that $n_{l} \leq n$. We have $L\left(X_{1}^{n}\right) \geq L\left(X_{1}^{n_{l}}\right)$ since $X_{1}^{n_{l}}$ is a substring of $X_{1}^{n}$, whereas

$$
k_{l}=\frac{1}{2} k_{l+1}=\frac{1}{2}\left(\log n_{l+1}\right)^{1 /(\beta+\epsilon)} \geq \frac{1}{2}(\log n)^{1 /(\beta+\epsilon)} .
$$

Hence $L\left(X_{1}^{n}\right) \geq \frac{1}{2}(\log n)^{1 /(\beta+\epsilon)}$ holds for all but finitely many $n$ almost surely. This completes the second proof of Theorem 7 . 


\section{Acknowledgment}

The author wishes to thank Jan Mielniczuk, Paweł Teisseyre, Ioannis Kontoyiannis, and anonymous reviewers for very helpful comments.

\section{References}

[1] A. de Luca, "On the combinatorics of finite words," Theor. Comput. Sci., vol. 218, pp. 13-39, 1999.

[2] R. Kolpakov and G. Kucherov, "Finding maximal repetitions in a word in linear time," in 40th Annual Symposium on Foundations of Computer Science, 1999, 1999, pp. 596-604.

[3] — , "On maximal repetitions in words," J. Discr. Algor., vol. 1, pp. 159$186,1999$.

[4] M. Crochemore and L. Ilie, "Maximal repetitions in strings," J. Comp. Syst. Sci., vol. 74, pp. 796-807, 2008.

[5] P. Erdős and A. Rényi, "On a new law of large numbers," J. Anal. Math., vol. 22, pp. 103-111, 1970.

[6] R. Arratia and M. S. Waterman, "The Erdös-Rényi strong law for pattern matching with a given proportion of mismatches," Ann. Probab., vol. 17, pp. 1152-1169, 1989.

[7] P. C. Shields, "String matching: The ergodic case," Ann. Probab., vol. 20, pp. 1199-1203, 1992.

[8] _ , "String matching bounds via coding," Ann. Probab., vol. 25, pp. 329-336, 1997.

[9] I. Kontoyiannis and Y. Suhov, "Prefixes and the entropy rate for long-range sources," in Probability, Statistics, and Optimization: A Tribute to Peter Whittle, F. P. Kelly, Ed. Wiley, 1994, pp. 89-98.

[10] Ł. Dębowski, "On the vocabulary of grammar-based codes and the logical consistency of texts," IEEE Trans. Inform. Theory, vol. 57, pp. 4589-4599, 2011.

[11] _ _ "Regular Hilberg processes: An example of processes with a vanishing entropy rate," 2015, http://arxiv.org/abs/1508.06158.

[12] _ - "Maximal repetitions in written texts: Finite energy hypothesis vs. strong Hilberg conjecture," Entropy, vol. 17, pp. 5903-5919, 2015.

[13] D. S. Ornstein and B. Weiss, "Entropy and data compression schemes," IEEE Trans. Inform. Theory, vol. 39, pp. 78-83, 1993.

[14] W. Szpankowski, "Asymptotic properties of data compression and suffix trees," IEEE Trans. Inform. Theory, vol. 39, pp. 1647-1659, 1993. 
[15] I. Kontoyiannis, P. H. Algoet, Y. M. Suhov, and A. J. Wyner, "Nonparametric entropy estimation for stationary processes and random fields, with applications to English text," IEEE Trans. Inform. Theory, vol. 44, pp. $1319-1327,1998$.

[16] Y. Gao, I. Kontoyiannis, and E. Bienenstock, "Estimating the entropy of binary time series: Methodology, some theory and a simulation study," Entropy, vol. 10, pp. 71-99, 2008.

[17] C. Shannon, "Prediction and entropy of printed English," Bell Syst. Tech. J., vol. 30, pp. 50-64, 1951.

[18] T. M. Cover and R. C. King, "A convergent gambling estimate of the entropy of English," IEEE Trans. Inform. Theory, vol. 24, pp. 413-421, 1978.

[19] P. F. Brown, S. D. Pietra, V. J. D. Pietra, J. C. Lai, and R. L. Mercer, "An estimate of an upper bound for the entropy of English," Comput. Linguist., vol. 18 , no. 1, pp. 31-40, 1983.

[20] P. Grassberger, "Data compression and entropy estimates by nonsequential recursive pair substitution," 2002, http://xxx.lanl.gov/abs/ physics/0207023

[21] F. Behr, V. Fossum, M. Mitzenmacher, and D. Xiao, "Estimating and comparing entropy across written natural languages using PPM compression," in Proceedings of Data Compression Conference 2003, 2003, p. 416.

[22] R. Takahira, K. Tanaka-Ishii, and Ł. Dębowski, "Entropy rate estimates for natural language - a new extrapolation of compressed large-scale corpora," Entropy, vol. 18, no. 10, p. 364, 2016.

[23] W. Hilberg, "Der bekannte Grenzwert der redundanzfreien Information in Texten - eine Fehlinterpretation der Shannonschen Experimente?" Frequenz, vol. 44, pp. 243-248, 1990.

[24] R. M. Gray, Entropy and Information Theory. Springer, 1990.

[25] A. Rényi, "On measures of entropy and information," in Proceedings of the Fourth Berkeley Symposium on Mathematical Statistics and Probability, Volume 1: Contributions to the Theory of Statistics, 1961, pp. 547-561.

[26] E. Arikan, "An inequality on guessing and its application to sequential decoding," IEEE Trans. Inform. Theory, vol. 42, pp. 99-105, 1996.

[27] S. Berens, "Conditional Rényi entropy," Master's thesis, Leiden University, 2013.

[28] M. H. F. Ko, "Renyi entropy and recurrence," Ph.D. dissertation, University of Southern California, 2012.

[29] F. Jelinek, Statistical Methods for Speech Recognition. The MIT Press, 1997. 
[30] C. D. Manning and H. Schütze, Foundations of Statistical Natural Language Processing. The MIT Press, 1999.

[31] R. Rosenfeld, "Two decades of statistical language modeling. Where do we go from here?" Proc. IEEE, vol. 88, pp. 1270-1278, 2000.

[32] H. W. Lin and M. Tegmark, "Critical behavior in physics and probabilistic formal languages," Entropy, vol. 19, p. 299, 2017.

[33] I. Kontoyiannis, "Asymptotic recurrence and waiting times for stationary processes," J. Theor. Probab., vol. 11, pp. 795-811, 1998.

[34] M. Kac, "On the notion of recurrence in discrete stochastic processes," Bull. Amer. Math. Soc., vol. 53, pp. 1002-1010, 1947.

[35] A. D. Wyner and J. Ziv, "Some asymptotic properties of entropy of a stationary ergodic data source with applications to data compression," IEEE Trans. Inform. Theory, vol. 35, pp. 1250-1258, 1989.

[36] E. G. Altmann, J. B. Pierrehumbert, and A. E. Motter, "Beyond word frequency: Bursts, lulls, and scaling in the temporal distributions of words," PLoS ONE, vol. 4, p. e7678, 2009.

[37] Ł. Dębowski, "Estimation of entropy from subword complexity," in Challenges in Computational Statistics and Data Mining, S. Matwin and J. Mielniczuk, Eds. Springer, 2016, pp. 53-70.

[38] — - "Consistency of the plug-in estimator of the entropy rate for ergodic processes," in 2016 IEEE International Symposium on Information Theory (ISIT), 2016, pp. 1651-1655.

[39] S. Janson, S. Lonardi, and W. Szpankowski, "On average sequence complexity," Theor. Comput. Sci., vol. 326, pp. 213-227, 2004.

[40] S. Ferenczi, "Complexity of sequences and dynamical systems," Discr. Math., vol. 206, pp. 145-154, 1999.

[41] I. Gheorghiciuc and M. D. Ward, "On correlation polynomials and subword complexity," Discr. Math. Theo. Comp. Sci., vol. AH, pp. 1-18, 2007.

[42] E. E. Ivanko, "Exact approximation of average subword complexity of finite random words over finite alphabet," Trud. Inst. Mat. Meh. UrO RAN, vol. 14, no. 4, pp. 185-189, 2008. 